\title{
A novel approach to glioma therapy using an oncolytic adenovirus with two specific promoters
}

\author{
FENG LIU ${ }^{1}$, KAYA XU ${ }^{1}$, HUA YANG ${ }^{1}$, YUMING LI $^{1}$, JIAN LIU $^{1}$, JIXIANG WANG ${ }^{1}$ and ZHIZHONG GUAN ${ }^{2}$ \\ ${ }^{1}$ Department of Neurosurgery, The Hospital Affiliated to Guizhou Medical University; ${ }^{2}$ Department of Molecular Biology, \\ The Laboratory of Molecular Biology, Guizhou Medical University, Guiyang, Guizhou 550000, P.R. China
}

Received January 21, 2017; Accepted November 7, 2017

DOI: $10.3892 / \mathrm{ol} .2017 .7684$

\begin{abstract}
Gliomas are the most common type of primary brain tumor in adults, where more than half of the cases are malignant, and the prognosis is poor. The early viral 1A (E1A) protein has been widely recognized to be essential for adenoviral replication and production of progeny virions in human cells, a process that is regulated by human telomerase reverse transcriptase. The p53 gene, as a tumor suppressor, regulates diverse cellular processes, including cell cycle arrest, cell autophagy, senescence and apoptosis. Dysfunction of the p53 pathways is common in malignant gliomas. Exogenous expression of p53 during adenovirus replication in human cancer cells may accelerate cell death and improve the release of early virus progeny. In the present study, a conditionally replicative adenovirus (CRAd) Ad-Tp-E1A-Gp-p53, which expressed functional p53 protein when replicating in cancer cells, was constructed. Next, the level of p53 expression in U251 cells was determined by western blot analysis, and the inhibitory effect of Ad-Tp-E1A-Gp-p53 on U251 cells was detected via an MTT assay. The results indicated that p53 expression was upregulated with an increase in the multiplicity of infection (MOI) of Ad-Tp-E1A-Gp-p53. Additionally, the inhibitory effects of Ad-Tp-E1A-Gp-p53 in different groups were significantly different $(\mathrm{P}<0.05)$, with the inhibition ratio of the experimental groups being higher, compared with the control group $(\mathrm{P}<0.05)$. Furthermore, the inhibition ratio increased with increases in the MOI of Ad-Tp-E1A-Gp-p53. Therefore, the expression of functional p53 and that of E1A may increase
\end{abstract}

Correspondence to: Dr Zhizhong Guan, Department of Molecular Biology, The Laboratory of Molecular Biology, Guizhou Medical University, 4 Beijing Road, Guiyang, Guizhou 550000, P.R. China E-mail: zhizhongguan1@yahoo.com

Abbreviations: E1A, early viral 1A; CRAd, conditionally replicative adenovirus; hTERT, human telomerase reverse transcriptase; GFAP, glial fibrillary acidic protein; CPE, cytopathic effect; SDS, sodium dodecyl sulfate

Key words: human telomerase reverse transcriptase, glial fibrillary acidic protein, early viral $1 \mathrm{~A}, \mathrm{p} 53$, conditionally replicative adenovirus the potency of CRAd, and overexpression of p53 through CRAd is a promising approach to more effective treatments in a number of human cancer types.

\section{Introduction}

Approximately 250,000 people worldwide are diagnosed with brain cancer annually. Unfortunately, only $20 \%$ of all brain tumors are of primary origin at the time of diagnosis, while $\sim 80 \%$ are metastatic (1). Gliomas are the most common primary brain tumor in adults and more than half are malignant (2). The current therapy for gliomas is multimodal, including surgical resection, radiotherapy and chemotherapy. However, due to the invasive properties of the cells, these treatment options are not always effective. Furthermore, the prognosis of patients with glioblastoma is poor and of invariable recurrence, with a median survival time of slightly $>1$ year $(3,4)$. In the search for alternative treatment modalities, oncolytic viruses have recently received increasingly widespread attention for their potential in novel cancer therapy $(5,6)$. Oncolytic viruses are effective for tumor therapy as they have the capacity to selectively replicate in tumor cells, to spread viral progeny in tumor tissues and to eventually induce tumor cell apoptosis (7). In this respect, conditionally replicative adenoviruses (CRAds) appear to be attractive anticancer agents and are currently being evaluated in clinical trials (8). The replication of CRAds occurs only in tumor cells and not in normal cells, which reflects the safety of this agent.

Conditionally replicative adenoviruses are designed to exert intrinsic anticancer activity through selective replication in cancer cells and to induce lysis in order to destroy these cells (9). Oncolytic viruses have been developed by adding specific promoter elements and therapeutic genes, including the human telomerase reverse transcriptase (hTERT) gene to control gene expression essential for adenoviral replication, and the p53 gene to promote the dissolution of tumor cells $(7,10)$. In addition, the release of viral progeny from infected tumor cells offers a potential to amplify the oncolytic effects of CRAds by lateral spread in a solid tumor $(6,9)$.

Self-replicated adenoviruses have previously been demonstrated to affect the progression of cancer cells in vitro by expressing p53. Promoters have been used previously to increase adenoviral replication or p53 expression, but the use of two promoters at same time is rare $(6,10)$. In the present 
study, to enhance the oncolysis of CRAd on tumor cells, a P74-Tp-Gp53 plasmid, containing the p53 gene, the early region $1 \mathrm{~A}$ (E1A) gene and two tumor-specific promoters hTERT and glial fibrillary acidic protein (GFAP), was constructed. The plasmid p74-Tp-Gp53 was restructured with the plasmid of an adenovirus skeleton, PPE3, into the recombinant oncolytic adenovirus, Ad-Tp-E1A-Gp-p53, to selectively replicate in tumor cells and to restrain cell growth by expressing the p53 gene. Therefore, this approach may serve as a promising therapeutic agent for the treatment of numerous types of cancer.

\section{Materials and methods}

Cell culture. The human glioma U251 and T98G cell lines, and the human embryonic lung MRC-5 cell line as well as the 293 cell line were cultured in Dulbecco's modified Eagle's medium (DMEM) plus GlutaMAX ${ }^{\mathrm{TM}}$ (Gibco; Thermo Fisher Scientific, Inc., Waltham, MA, USA), supplemented with $10 \%$ heat-inactivated fetal bovine serum (FBS; Gibco; Thermo Fisher Scientific, Inc.), $100 \mathrm{U} / \mathrm{ml}$ penicillin and $100 \mathrm{~g} / \mathrm{ml}$ streptomycin at $37^{\circ} \mathrm{C}$ in $5 \% \mathrm{CO}_{2}$.

Plasmid construction. hTERT and GFAP were designed according to the sequences published in GenBank (https://www.ncbi.nlm.nih.gov/genbank/; accession numbers, NM_198253 and M67446, respectively), and were synthesized by Generay Biotech Co., Ltd. (Shanghai, China). Next, restriction sites, BgIII (5'-AGATCT-3') and HindIII (5'-AAGCTT-3'), were added upstream and downstream of hTERT. Subsequently, hTERT and GFAP were cloned into the Dual-Luciferase ${ }^{\circledR}$ Reporter vector, pGL3-Basic (empty vector; Promega Corporation, Madison, WI, USA), resulting in the plasmids pGL3-hTERTp and pGL3-GFAP, respectively. Using Lipofectamine ${ }^{\circledR} 2000$ (Invitrogen; Thermo Fisher Scientific, Inc.), plasmids were transfected into the MRC-5 (11), U251 and T98 G cells for $2 \mathrm{~h}$. A total of $24 \mathrm{~h}$ after transfection, the activity of the promoters was detected using the Dual-Luciferase reporter gene detection system (Invitrogen; Thermo Fisher Scientific, Inc.). Cells in the normal control group remained intact and pRL-TK (Promega Corporation) was used as a control reporter vector, and was used in combination with any experimental reporter vector to co-transfect mammalian cells. Renilla promoters (Promega Corporation) were co-transfected as an internal control. Firefly luciferase activity was normalized to renilla luciferase activity for individual analysis.

To enhance the activity of pGL3-GFAP, four optimized HIF-binding site optHBS enhancer sites (5'-TACGTGCAG TACGTGCAGTACGTGCAGTACGTGCAG-3') were cloned (Invitrogen; Thermo Fisher Scientific, Inc.) into $M l u \mathrm{I} / \mathrm{XhoI}$ sites of pGL3-GFAP, resulting in the plasmid pGL3-ENGFAP. pGL3-ENGFAP was then identified by excising with two different double enzyme systems ( $M l u \mathrm{I} / \mathrm{XhoI}$ and $P v u \mathrm{I} / P v u \mathrm{II})$ and the excision product was detected by $2 \%$ agarose gel electrophoresis.

A 1,836 bp fragment was obtained from the double digestion of pGL3-ENGFAP with MluI/HindIII followed by EcoRI/XbaI and cloned into the EcoRI/XbaI sites of PCA19 to generate the plasmid PCA19-ENGFAP. Subsequently, two different enzyme systems (EcoRI/XbaI and PstI) and 2\% agarose gel electrophoresis were used to verify the generation of PCA19-ENGFAP. Next, the early viral1A (E1A) gene was cloned into NcoI/SalI sites of pGL3-hTERT, and the plasmid pGL3-hTERT-E1A (P74-Tp) was generated. The p53 gene was excised by double digestion of PENTER15-p53 (Invitrogen; Thermo Fisher Scientific, Inc.) with SalI and EcoRI and was cloned into the SalI and EcoRI sites in PCA19-ENGFAP, resulting in the plasmid PCA19-Gp53. The plasmid PCA19-Gp53 was excised by digestion with EcoRI, SalI and $P V U$ II, and the excised product was detected by $2 \%$ agarose gel electrophoresis. Additionally, the 3,765 bp fragment excised from PCA19-Gp53 using BglII was cloned into the 8,465 bp fragment excised from pGL3-hTERT-E1A (P74-TP) using $B g l \mathrm{II}$, to yield the plasmid p74-Tp-Gp53. A different enzyme system (BamHI and HindIII; Invitrogen; Thermo Fisher Scientific, Inc.) was used to identify the p74-Tp-Gp53 plasmid prior to detection of the excised fragments by $2 \%$ agarose gel electrophoresis.

Recombination of oncolytic adenovirus. The shuttle plasmid p74-Tp-Gp53 and the adenovirus vector PPE3 (both Invitrogen; Thermo Fisher Scientific, Inc.) were co-transfected into well-cultured 293 cells using Lipofectamine ${ }^{\circledR} 2000$ (Invitrogen; Thermo Fisher Scientific, Inc.) to complete the virus recombination. Following 9 days of incubation at $37^{\circ} \mathrm{C}$, virus plaques were observable and were purified three times. Subsequently, 293 cells were alternately frozen and thawed to collect the restructured oncolytic adenovirus, Ad-Tp-E1A-Gp-p53. Polymerase chain reaction was then performed to determine the recombination of the Ad-Tp-E1A-Gp-p53 oncolytic adenovirus.

DNA extraction and PCR. Genomic DNA of the oncolytic adenovirus, Ad-Tp-E1A-Gp-p53, was extracted using a Blood Genome DNA Extraction kit (Takara Biotechnology Co., Inc., Dalian, China) according to the manufacturer's protocol and was stored at $-80^{\circ} \mathrm{C}$. To determine the recombination sites of the oncolytic adenovirus, PCR was performed with repeated three times using the following primers: GT154+GT156 forward, 5'-CCCACCGGTCACAGACGCCCAGGAC-3' and reverse, 5'-GTGGCCGGGGCCAGGGCTTCCC-3'; W267+W268 forward, 5'-CCGGACGATATTGAACAATGG TTC-3' and reverse, 5'-GTGAAATATTCTCCATCCAGT GG-3'; and W331+W332 forward, 5'-CGACGCGTCCCT CTAGATACGTGCAGTACGTGCAGTACGTGCAGTACG TGCAGAA-3' and reverse, 5'-CCGCTCGAGTTCCCACAC ATCAGCCTGGAGAGAT-3' to amplify hTERT, p53 and GFAP, respectively. The PCR reaction was set at an initial denaturation step of $3 \mathrm{~min}$ at $95^{\circ} \mathrm{C}$ followed by 35 cycles of $95^{\circ} \mathrm{C}$ for $40 \mathrm{sec}, 58^{\circ} \mathrm{C}$ for $40 \mathrm{sec}, 72^{\circ} \mathrm{C}$ for $90 \mathrm{sec}$ and, finally, $72^{\circ} \mathrm{C}$ for $10 \mathrm{~min}$. The PCR products were subsequently detected by $2 \%$ agarose gel electrophoresis.

Detection of viral titer by $50 \%$ cell culture infection dose (TCID50). 293 cells (permissive to viral infection) were seeded onto 96 -well plates $\left(10^{4}\right.$ cells/well). After $24 \mathrm{~h}$, eight serial 10-fold dilutions of freeze/thaw lysates from infected cells were seed into a 96-well plate (100 $\mu \mathrm{l} /$ well). After 10 days of incubation at $37^{\circ} \mathrm{C}$, the observable cytopathic effect (CPE) 

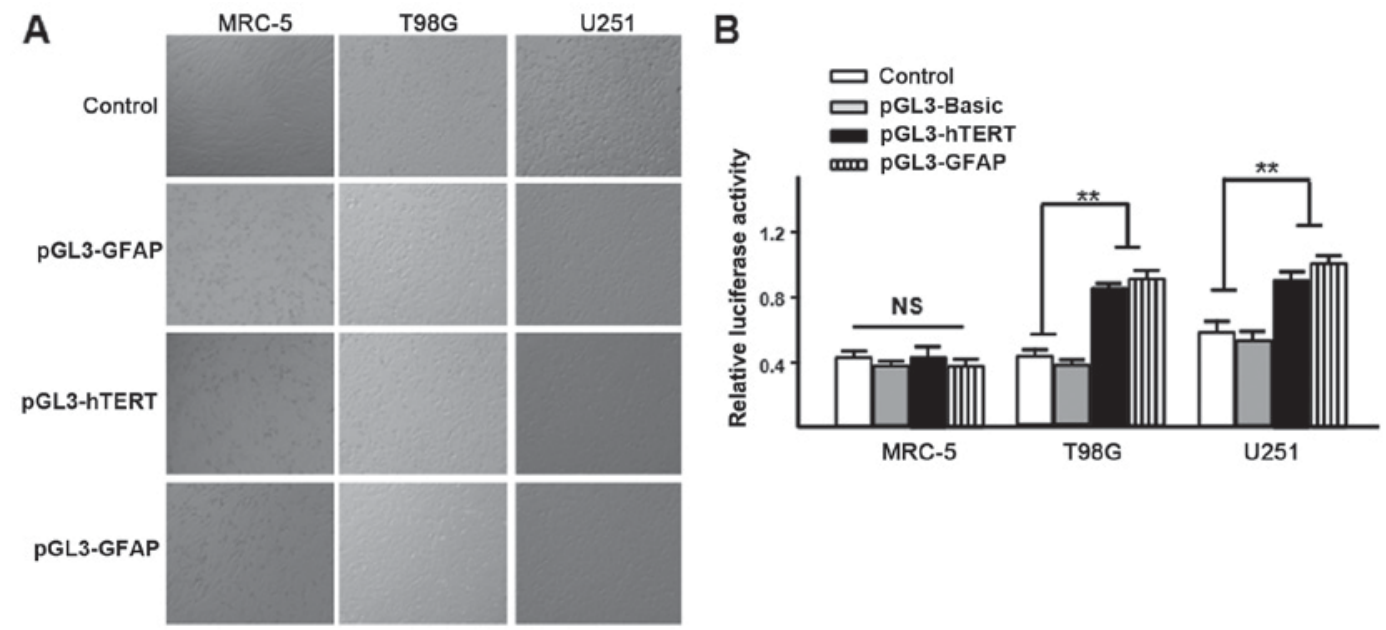

Figure 1. Activity of pGL3-hTERTp and pGL3-GFAP in different cells. (A) The plasmids (pGL3-hTERTp and pGL3-GFAP) were transfected into the cultured MRC-5 (11), U251 and T98 G cells (magnification, x100). (B) The relative luciferase activity of pGL3-hTERTp and pGL3-GFAP was detected using a dual-luciferase reporter assay. Data are presented as the mean \pm standard deviation $(n=3) .{ }^{* *} \mathrm{P}<0.01$. NS, not significant. hTERT, human telomerase reverse transcriptase; GFAP, glial fibrillary acidic protein.

per dilution was counted, and the ratio between infected and unaffected wells was determined. The viral titer was then calculated using the following equation: TCID50 $=10^{\mathrm{L}+\mathrm{d}(\mathrm{s}-0.5)}$, where $\mathrm{L}$ is the $\log$ of the lowest dilution (e.g., If $10^{-1}$ is the minimum dilution degree, $\mathrm{L}=1$ ), $\mathrm{d}$ is the dilution coefficient (e.g., If there is a 10 -fold dilution, $\mathrm{d}=1$ ), and $\mathrm{s}$ is the sum of the CPE rate observed at each dilution.

Western blot analysis. U251 cells were infected with Ad-Tp-E1A-Gp-p53 at different multiplicities of infection (MOIs; 0, 1, 10, 100 and 1,000). A bicinchoninic acid assay was used to determine protein concentrations. After $48 \mathrm{~h}$ of incubation, infected U251 cells were lysed using lysis buffer [0.125 M TRIS- $\mathrm{HCl}$ (pH 6.8), $2 \%$ SDS and proteinase inhibitor; Abcam, Cambridge, UK]. Total protein $(20 \mu \mathrm{g} /$ lane $)$ was then separated on a SDS polyacrylamide gel $(12.5 \%)$ and blotted onto HyBond N membranes (EMD Millipore, Billerica, MA, USA). Following blocking with a 5\% skimmed milk solution in TBS with $0.1 \%$ Tween- 20 for $2 \mathrm{~h}$ at room temperature, adenoviral p53 expression was detected using the following primary antibodies: Anti-p53 [DO-1] (cat. no. ab1101; dilution, 1:1,000; Abcam) and anti-GAPDH [6C5] (cat. no. ab8245; dilution, 1:2,000; Abcam), and incubated for $12 \mathrm{~h}$ at $4^{\circ} \mathrm{C}$. GAPDH (cat. no. ab8245; dilution, 1:2,000; Abcam) served as a loading control. Membranes were then incubated with a horseradish peroxidase-conjugated goat anti-mouse immunoglobulin $\mathrm{G}$ secondary antibody (cat. no. ab6789; dilution, 1:5,000; Abcam) for $2 \mathrm{~h}$ at room temperature. The antigen-antibody complexes were visualized using an enhanced chemiluminescence detection plus kit (cat. no. PE0010; EMD Millipore), according to the manufacturer's protocol.

Detection of the growth inhibiting effect of Ad-Tp-E1A-Gp-p53 on U251 cells by MTT. Tumor U251 cells were seeded onto 96 -well plates $\left(10^{4}\right.$ cells/well $)$ and were infected with freeze/thaw lysates of infected cells at different MOIs $(0,1,10,100$ and 1,000$)$ following incubation for $24 \mathrm{~h}$ at $37^{\circ} \mathrm{C}$ (Control, 0 MOI; Group 1, 1 MOI; group 2, 10 MOI; group 3,
$100 \mathrm{MOI}$; group 4, 1,000 MOI). After $2 \mathrm{~h}$, the culture solution was discarded and 5\% FBS/DMEM was added. After incubation for $72 \mathrm{~h}$ at $37^{\circ} \mathrm{C}$, MTT $(5 \mathrm{mg} / \mathrm{ml})$ was added to the 96-well plates $(20 \mu \mathrm{l} /$ well). After $4 \mathrm{~h}, 200 \mu \mathrm{l}$ dimethyl sulfoxide was added to the 96-well plates, and then the 96-well plates were agitated to dissolve the purple formazan. Subsequently, optical density (OD) values were measured at $490 \mathrm{~nm}$. The cell growth inhibition rate was calculated using the following equation: Inhibition rate $(\%)=($ control group OD value-experimental group OD value)/control group OD value.

Statistical analysis. All analyses were performed using SPSS software package (version 19.0; IBM Corp., Armonk, NY, USA). Statistical differences between all groups (experimental groups and the control group) were compared using one-way analysis of variance, followed by the Student-Newman-Keuls post hoc test. Data are expressed as the mean \pm standard deviation of three independent experiments. $\mathrm{P}<0.05$ was considered to indicate a statistically significant difference.

\section{Results}

Activity of $p$ GL3-hTERTp and pGL3-GFAP. The plasmids, pGL3-hTERTp and pGL3-GFAP, were transfected into the MRC-5, U251 and T98 G cells with pRL-TK (Invitrogen; Thermo Fisher Scientific, Inc.) at a ratio of 50:1, respectively. After $24 \mathrm{~h}$ of incubation, the activity was detected using a dual-luciferase reporter assay. It was observed that the hTERT promoter is active in U251 and T98G glioma cells, but it is not active in human MRC- 5 cells, and the same finding was obtained for the GFAP promoter (Fig. 1). These results indicate that there is a good level of specificity of the hTERT and GFAP promoters to glioma cells.

Verification of pGL3-ENGFAP, PCA19-ENGFAP, PCA19-Gp53, P74-Tp-Gp53 plasmids and the recombinant adenovirus Ad-Tp-E1A-Gp-p53. The results of agarose gel electrophoresis revealed that two predicted fragments were 

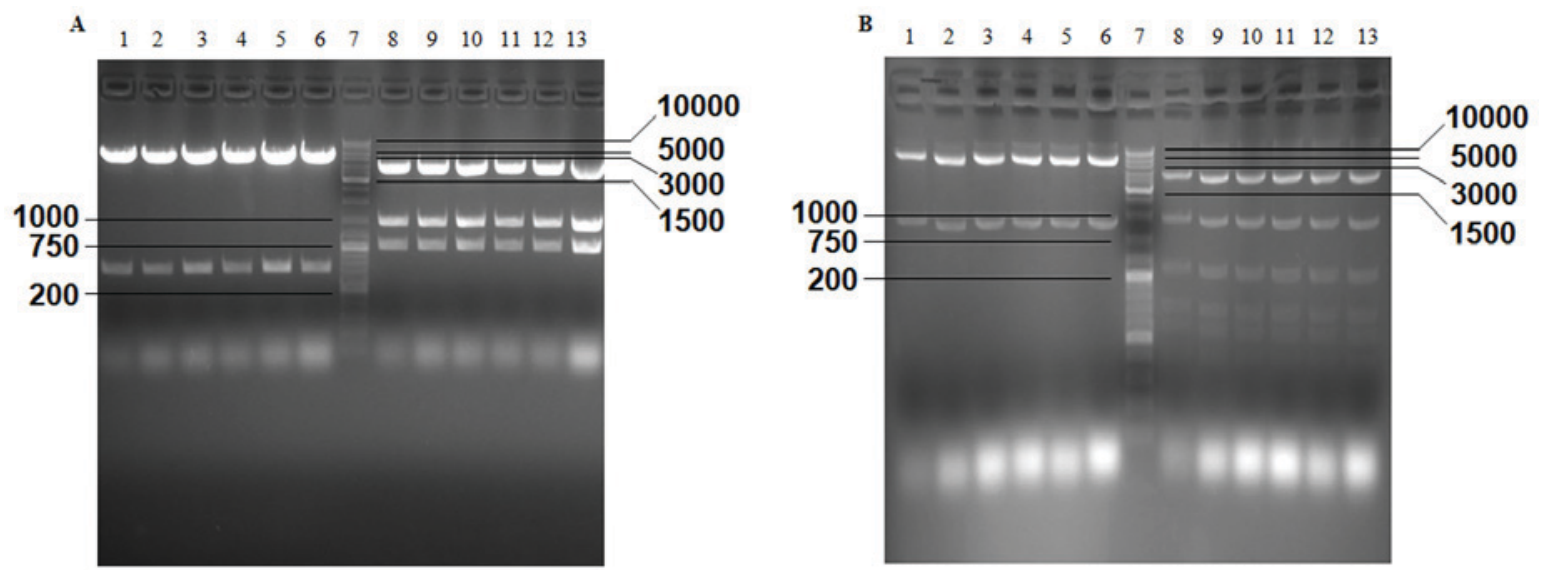

Figure 2. Verification of pGL3-ENGFAP and PCA19-ENGFAP plasmids by gel electrophoresis, according to the size (bp) of excised fragments. (A) Excised fragments of pGL3-ENGFAP were detected by gel electrophoresis. Lanes 1-6, double digestion with MluI and XhoI; lane 7, GeneRuler ${ }^{\mathrm{TM}}$ DNA Ladder mix (10,000 bp); and lanes 8-13, double digestion with $P v u \mathrm{I}$ and PvuII. (B) Excised fragments of PCA19-ENGFAP were detected by gel electrophoresis. Lanes 1-6, double digestion with EcoRI and XbaI; lane 7, GeneRuler ${ }^{\mathrm{TM}}$ DNA Ladder Mix; and lanes 8-13, digestion with PstI. GFAP, glial fibrillary acidic protein.
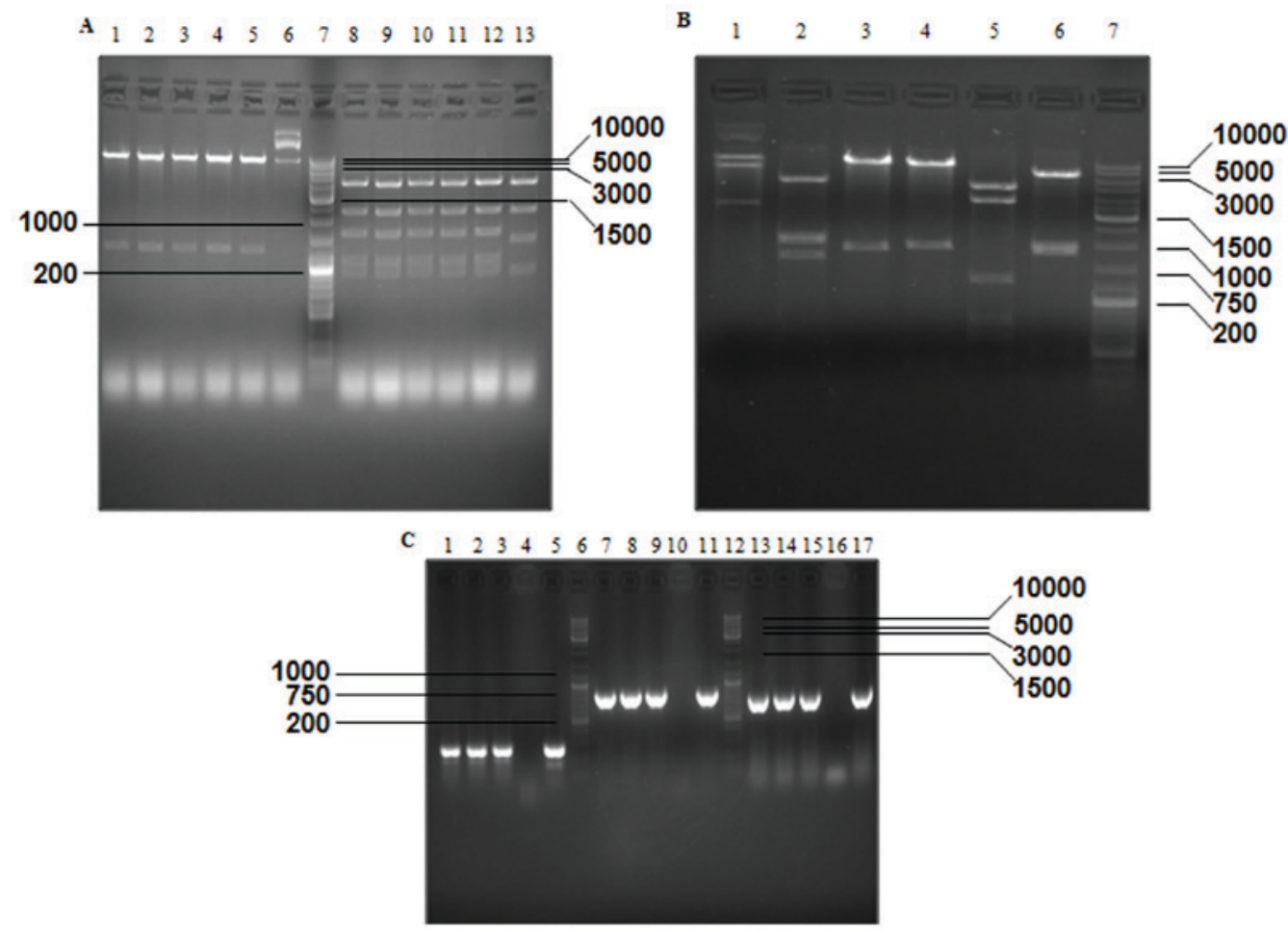

Figure 3. Verification of PCA19-Gp53 and p74-Tp-Gp53 plasmids by gel electrophoresis, according to the size (bp) of excised fragments. (A) Excised fragments of PCA19-Gp53 detected by gel electrophoresis. Lanes 1-6, double digestion with EcoRI and SalI; lane 7, GeneRuler ${ }^{\mathrm{TM}}$ DNA Ladder mix (10,000 bp); lanes 8-13, digestion with $P v u I I$. (B) Excised fragments of P74-Tp-Gp53 detected by gel electrophoresis. Lanes 1-6, digestion of PCA19-Gp53 by BglII, SacI and XbaI, NcoI and SalI, BamHI, PstI and NcoI, respectively; and lane 7, GeneRuler ${ }^{\mathrm{TM}}$ DNA Ladder Mix (10,000 bp). (C) DNA fragments of Ad-Tp-E1A-Gp-p53 were detected by gel electrophoresis. Lanes 1-3, amplification of Ad-Tp-E1A-Gp-p53 using the GT154+GT156 primer; lanes 4, 10 and 16, negative control; lane 5, amplification of plasmid P74-TP using the GT154+GT156 primer; lanes 6 and 12, GeneRuler ${ }^{\mathrm{TM}}$ DNA Ladder Mix; lanes 7-9, amplification of Ad-Tp-E1A-Gp-p53 using the W267+W268 primer; lane 11, amplification of plasmid PENTER-p53 using the W267+W268 primer; lanes 13-15, amplification of Ad-Tp-E1A-Gp-p53 using the W331+W332 primer; and lane 17, amplification of plasmidpGL3-GFAP by using the W331+W332 primer. E1A, early viral 1A; GFAP, glial fibrillary acidic protein.

obtained from pGL3-ENGFAP, which was double digested with $M l u \mathrm{I}$ and $\mathrm{XhoI}$, and three fragments were obtained from the double digestion of pGL3-ENGFAP with $P v u \mathrm{I}$ and $P v u \mathrm{II}$ (Fig. 2A). Following double digestion of PCA19-ENGFAP with EcoRI and $X b a \mathrm{I}$ and digestion with PstI, two and seven predicted fragments were obtained (Fig. 2B). EcoRI+SalI and $P v u$ II were used to identify PCA19-Gp53, and two and seven predicted fragments were generated, respectively (Fig. 3A). As a result of the excision with BglII, SacI+XbaI, NcoI+SalI, BamHI, PstI and NcoI, different numbers of predicted fragments $(2,5,6,4,7$ and 5 , respectively) were obtained from p74-Tp-Gp53 (Fig. 3B). Additionally, the results demonstrated that partial fragments of TERTp (size, 266 bp), p53 (size, 847 bp) and GFAP (size, 744 bp) were 
successfully amplified from Ad-Tp-E1A-Gp-p53 using PCR (Fig. 3C).

Viral titer of $p 74-T p-G p 53$. Using the aforementioned equation for TCID50 $=10^{\mathrm{L}+\mathrm{d}(\mathrm{s}-0.5)}$, a viral titer of $4.15 \times 10^{11} \mathrm{PFU} / \mathrm{ml}$ Ad-Tp-E1A-Gp-p53 was calculated.

Expression of functional p53 in cancer cells infected with adenovirus. To investigate whether Ad-Tp-E1A-Gp-p53 affects the expression of $\mathrm{p} 53$, western blot analysis was performed and the levels of 553 expression in the Ad-Tp-E1A-Gp-p53-infected U251 cells at different MOIs were compared. As indicated in Fig. 4A, infection with Ad-Tp-E1A-Gp-p53 was able to upregulate p53 expression in U251 cells, and the level of p53 expression gradually increased with an increase in MOI.

Inhibitory effect of Ad-Tp-E1A-Gp-p53 on U251 cell growth. To evaluate the efficacy of viral transmission and the therapeutic potential of Ad-Tp-E1A-Gp-p53 in vitro, U251 cells derived from human brain gliomas were infected with Ad-Tp-E1A-Gp-p53 at different MOIs and the inhibition rate was calculated (Fig. 4B). The inhibitory effect of different MOIs of Ad-Tp-E1A-Gp-p53 was significantly different $(\mathrm{P}<0.05)$, in which the inhibition ratios of the experimental groups 1-4 were significantly higher compared with the control group $(\mathrm{P}<0.05)$. Additionally, the inhibition ratio of the four experimental groups exhibited an increasing trend as the MOI of Ad-Tp-E1A-Gp-p53 increased.

\section{Discussion}

In the present study, pGL3-hTERTp and pGL3-GFAP plasmids were successfully constructed and due to low activity, four optHBS enhancer sites were cloned into pGL3-GFAP. Additionally, a recombinant p74-Tp-Gp53 plasmid was constructed to specifically express E1A and p53 in tumor cells. Furthermore, Ad-Tp-E1A-Gp-p53 was demonstrated to suppress the growth of U251 cells through the expression of functional p53.

With the development of molecular biology and in-depth knowledge of viral gene function, it has become possible to genetically re-engineer viruses, and these may be used to selectively target tumor cells through the use of adenoviruses and herpesviruses $(12,13)$. Conditionally replicative adenoviruses (CRAds) have recently presented as novel agents for cancer therapy and the use of these CRAds has been evaluated in preclinical trials (14). These evaluations have already demonstrated the potential of these adenoviruses in the therapy of malignant brain tumors. For example, the conditionally replicative adenovirus, ONYX-015, has entered into clinical trials for malignant glioma $(6,15,16)$. The release and spread of conditionally replicative adenoviruses progeny depend on the replication efficiency of the adenovirus in cancer cells and their oncolytic capacity to induce death in cancer cells at the late stages of infection (9).

Since the E1A protein has been identified and widely recognized to be essential for adenoviral replication and the production of progeny virions in human cells, a number of telomerase promoter-regulated adenoviral vectors retain E1A genes (17). Additionally, the catalytic component of hTERT is

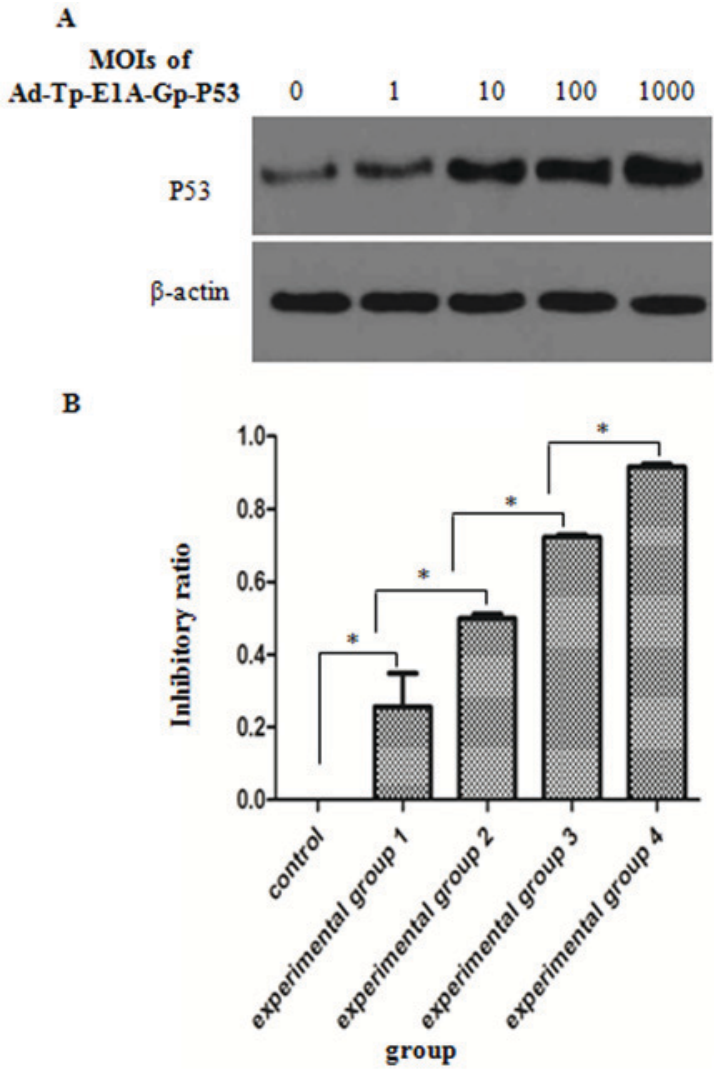

Figure 4. Adenovirus Ad-Tp-E1A-Gp-p53 suppresses the growth of U251 cells. (A) Quantitative analysis of p53 expression via western blot analysis. $\beta$-actin gene was used as an internal control. (B) Inhibitory effect of Ad-Tp-E1A-Gp-p53 on the growth of U251 cells as detected by MTT. Data are presented as the mean \pm standard deviation $(n=3)$. ${ }^{*} \mathrm{P}<0.05$. Group 1 , $1 \mathrm{MOI}$; group 2, $10 \mathrm{MOI}$; group 3, $100 \mathrm{MOI}$; group 4, 1,000 MOI. MOI, multiplicity of infection. E1A, early viral $1 \mathrm{~A}$.

not expressed in the majority of primary somatic human cells, whereas major cancer cells are able to reactivate telomerase by transcriptional upregulation of hTERT (18). Furthermore, the hTERT gene, as a promoter, controls the gene expression that is essential for adenoviral replication (19). Therefore, hTERT is usually used to induce E1A expression and adenovirus replication in tumor cells. In the present study, hTERT was cloned together with the E1A gene into pGL3-Basic to induce E1A expression in tumor cells. GFAP, which was used in the present study as a promoter, has been previously demonstrated to be able to promote p53 expression and is downregulated in glioblastoma cells $(20,21)$. GFAP and hTERT have been demonstrated to be glioma specific and are not expressed in MRC-5 cells (11), and these findings were confirmed in the present study.

The p53 gene, as a tumor suppressor, regulates diverse cellular processes, including cell cycle arrest, cell autophagy, senescence and apoptosis (22). In normal cells, the interaction between cellular proteins and encoded proteins, including cellular p53 proteins with viral E1A proteins, is necessary to complete the viral life cycle. However, when this interaction is dysregulated, normal cells gain the ability to develop into tumor cells $(9,23)$.

The p53 suppressor protein is one component of the cell apoptosis pathway that is exploited by adenoviruses. Unfortunately, p53 is frequently inactivated by genetic 
alterations in $\sim 50 \%$ of all types of human cancer. For example, dysfunction of the p53 signaling pathway is common in malignant gliomas, which leads to a non-functional p53 pathway and inhibition of CRAd-induced cell death $(6,24)$.

It was reported in a previous study that p53 expression was higher in high-grade brain glioma compared with low-grade brain glioma $(\mathrm{P}<0.05)$, and the expression was not associated with patient sex or age, or the size of the tumor, demonstrating that p53 serves an important role in the occurrence and development of brain glioma (25). Therefore, restoring the function of wild-type p53 in p53-inactivated tumor cells is a potential treatment for certain types of tumor. In recent years, adenovirus-mediated p53 gene therapy has been rapidly developed as a promising antitumor strategy and has been employed in a number of preclinical experiments and clinical studies $(22,25,26)$. In this regard, p53 is a common gene inserted in CRAd to control tumor cell growth and to cause cell apoptosis.

A number of experiments involving the use of p53 in CRAd have been performed. For example, Chen et al (26) demonstrated that p53-induced microRNA-107 inhibited brain tumor cell growth, which indicates that it serves as a tumor suppressor and thus, may be used as a target for glioma therapy. Additionally, Yang et al (27) suggested a combination of recombinant adenovirus-p53 (rAd-p53) with fractionated stereotactic radiotherapy (fSRT) is an effective and relatively safe method for the treatment of primary hepatocellular carcinoma (HCC) compared with fSRT monotherapy, indicating that rAd-p53 combined with fSRT may be preferred as a local method to treat primary HCC if the patients are unable to undergo surgery or refuse operation.

In the present study, a conditionally replicating adenovirus (Ad-Tp-E1A-Gp-p53) was constructed using the two plasmids pGL3-hTERT-E1A (p74-Tp) and PCA19-Gp53. Next, the expression level of functional p53 in adenovirus-infected $\mathrm{U} 251$ cells was detected by western blot analysis, and the results revealed that the expression of p53 was upregulated with an increasing MOI. Additionally, the inhibitory effect of Ad-Tp-E1A-Gp-p53 on U251 cells was detected by MTT and the results indicated that the inhibitory effects of Ad-Tp-E1A-Gp-p53 in different experimental groups (with different MOIs) were significantly different $(\mathrm{P}<0.05)$, with the inhibition ratio of the experimental groups being higher compared with the control group $(\mathrm{P}<0.05)$. Furthermore, the inhibition ratio increased with increases in MOI, indicating that the expression of functional p53 enhances the inhibitory effect of Ad-Tp-E1A-Gp-p53 on U251 cells.

In conclusion, in the present study, p74-Tp, PCA19-Gp53 and Ad-Tp-E1A-Gp-p53 were constructed. It was also demonstrated that hTERT and GFAP were able to promote the expression of their downstream genes in vitro. Furthermore, Ad-Tp-E1A-Gp-p53 was able to suppress the growth of U251 cells and the inhibitory effect increased with increasing MOIs of Ad-Tp-E1A-Gp-p53. Therefore, Ad-Tp-E1A-Gp-p53 may contribute to more effective treatment for different types of human cancer to enhance the potency of CRAd through the expression of functional p53. Although the present study demonstrated that the constructed CRAd represses the growth of glioma cells, there were certain limitations. The human MRC-5 cell line was used rather than normal glial cells to verify glioma specificity of the constructed virus and thus, future experiments should use normal glial cells as a control to further verify the effectiveness of the constructed virus.

\section{Acknowledgements}

The authors would like to thank Dr Qijun Qian and Dr Hongping Wu from The Second Military Medical University (Shanghai, China) for providing technical assistance during the experiments. The present study was supported by the Science and Technology Mutual Fund of Guizhou Province (grant no. [2014] 7116) and the Science and Technology Fund of Guizhou Health and Family Planning Commission (grant no. gzwjkj 2014-2-144).

\section{References}

1. Ulasov I, Borovjagin AV, Kaverina N, Schroeder B, Shah N, Lin B, Baryshnikov A and Cobbs C: MT1-MMP silencing by an shRNA-armed glioma-targeted conditionally replicative adenovirus (CRAd) improves its anti-glioma efficacy in vitro and in vivo. Cancer Lett 365: 240-250, 2015.

2. Gilbert MR, Dignam JJ, Armstrong TS, Wefel JS, Blumenthal DT, Vogelbaum MA, Colman H, Chakravarti A, Pugh S, Won M, et al: A randomized trial of bevacizumab for newly diagnosed glioblastoma. N Engl J Med 370: 699-708, 2014.

3. Westphal M, Ylä-Herttuala S, Martin J, Warnke P, Menei P, Eckland D, Kinley J, Kay R and Ram Z; ASPECT Study Group: Adenovirus-mediated gene therapy with sitimagene ceradenovec followed by intravenous ganciclovir for patients with operable high-grade glioma (ASPECT): a randomised, open-label, phase 3 trial. Lancet Oncol 14: 823-833, 2013.

4. Holland EC: Glioblastoma multiforme: The terminator. Proc Natl Acad Sci USA 97: 6242-6244, 2000.

5. Nemunaitis J and Edelman J: Selectively replicating viral vectors. Cancer Gene Ther 9: 987-1000, 2002.

6. Geoerger B, Vassal G, Opolon P, Dirven CM, Morizet J Laudani L, Grill J, Giaccone G, Vandertop WP, Gerritsen WR and van Beusechem VW: Oncolytic activity of p53-expressing conditionally replicative adenovirus AdDelta24-p53 against human malignant glioma. Cancer Res 64: 5753-5759, 2004.

7. Wei F, Wang H, Chen X, Li C and Huang Q: Dissecting the roles of $\mathrm{E} 1 \mathrm{~A}$ and $\mathrm{E} 1 \mathrm{~B}$ in adenoviral replication and RCAd-enhanced RDAd transduction efficacy on tumor cells. Cancer Biol Ther 15: 1358-1366, 2014.

8. Alemany R, Balagué C and Curiel DT: Replicative adenoviruses for cancer therapy. Nat Biotechnol 18: 723-727, 2000.

9. van Beusechem VW, van den Doel PB, Grill J, Pinedo HM and Gerritsen WR: Conditionally replicative adenovirus expressing p53 exhibits enhanced oncolytic potency. Cancer Res 62: 6165-6171, 2002

10. Fukuda K, Abei M, Ugai H, Kawashima R, Seo E, Wakayama M, Murata T, Endo S, Hamada H, Hyodo I and Yokoyama KK: E1A, E1B double-restricted replicative adenovirus at low dose greatly augments tumor-specific suicide gene therapy for gallbladder cancer. Cancer Gene Ther 16: 126-136, 2009.

11. Xu J, Mo Y, Wang X, Liu J, Zhang X, Wang J, Hu L, Yang C, Chen L and Wang Y: Conditionally replicative adenovirus-based mda-7/IL-24 expression enhances sensitivity of colon cancer cells to 5-fluorouracil and doxorubicin. J Gastroenterol 48: 203-213, 2013.

12. Chu RL, Post DE, Khuri FR and Van Meir EG: Use of replicating oncolytic adenoviruses in combination therapy for cancer. Clin Cancer Res 10: 5299-5312, 2004.

13. Chiocca EA: Oncolytic viruses. Nature Rev Cancer 2: 938-950, 2002.

14. Fueyo J, Alemany R, Gomez-Manzano C, Fuller GN, Khan A, Conrad CA, Liu TJ, Jiang H, Lemoine MG, Suzuki K, et al: Preclinical characterization of the antiglioma activity of a tropism-enhanced adenovirus targeted to the retinoblastoma pathway. J Natl Cancer Inst 95: 652-660, 2003. 
15. Geoerger B, Grill J, Opolon P, Morizet J, Aubert G, Terrier-Lacombe MJ, Bressac De-Paillerets B, Barrois M, Feunteun J, Kirn DH and Vassal G: Oncolytic activity of the E1B-55 kDa-deleted adenovirus ONYX-015 is independent of cellular p53 status in human malignant glioma xenografts. Cancer Res 62: 764-772, 2002.

16. Lamfers ML, Grill J, Dirven CM, Van Beusechem VW, Geoerger B, Van Den Berg J, Alemany R, Fueyo J, Curiel DT, Vassal G, et al: Potential of the conditionally replicative adenovirus Ad5-Delta24RGD in the treatment of malignant gliomas and its enhanced effect with radiotherapy. Cancer Res 62: 5736-5742, 2002.

17. Wirth T, Kuhnel F and Kubicka S: Telomerase-dependent gene therapy. Curr Mol Med 5: 243-251, 2005.

18. Wirth T, Zender L, Schulte B, Mundt B, Plentz R, Rudolph KL, Manns M, Kubicka S and Kühnel F: A telomerase-dependent conditionally replicating adenovirus for selective treatment of cancer. Cancer Res 63: 3181-3188, 2003.

19. Alemany R: Conditionally replicating adenoviruses for cancer treatment. Cancer Gene Ther: 235-248, 2005.

20. Wilhelmsson U, Eliasson C, Bjerkvig R and Pekny M: Loss of GFAP expression in high-grade astrocytomas does not contribute to tumor development or progression. Oncogene 22: 3407-3411, 2003.
21. Lee K, Jeon K, Kim JM, et al: Downregulation of GFAP, TSP-1 and p53 in human glioblastoma cell line, U373MG, by IE1 protein from human cytomegalovirus. Glia 51: 1-12, 2005.

22. Tazawa H, Kagawa $\mathrm{S}$ and Fujiwara T: Advances in adenovirus-mediated p53 cancer gene therapy. Expert Opin Biol Ther 13: 1569-1583, 2013.

23. Heise C,Hermiston T,Johnson L, Brooks G,Sampson-Johannes A, Williams A, Hawkins L and Kirn D: An adenovirus E1A mutant that demonstrates potent and selective systemic anti-tumoral efficacy. Nat Med 6: 1134-1139, 2000.

24. Collins V: Gene amplification in human gliomas. Glia 15: 289-296, 1995.

25. Lin T, Wang M, Liang HS and Liu EZ: The expression of p53, mgmt and egfr in brain glioma and clinical significance. J Biol Regul Homeost Agents 29: 143-149, 2015

26. Chen L, Zhang R, Li P, Liu Y, Qin K, Fa ZQ, Liu YJ, Ke YQ and Jiang XD: p53-induced microRNA-107 inhibits proliferation of glioma cells and down-regulates the expression of CDK6 and Notch-2. Neurosci Lett 534: 327-332, 2013.

27. Yang ZX, WangD, Wang G, Zhang QH, Liu JM, Peng $P$ and Liu XH: Clinical study of recombinant adenovirus-p53 combined with fractionated stereotactic radiotherapy for hepatocellular carcinoma. J Cancer Res Clin Oncol 136: 625-630, 2010. 